\title{
Evaluating Improvement in the Care of Depressed Elderly Patients: An Empirical Approach to the WHO Patient Safety Model
}

\author{
Elisabeth Severinsson ${ }^{*}$, Anne Lise Holm² \\ ${ }^{1}$ Centre for Women's, Family and Child Health, Faculty of Health Sciences, Buskerud \& Vestfold University \\ College, Kongsberg, Norway \\ ${ }^{2}$ Department of Health Science, Stord/Haugesund University College, Haugesund, Norway \\ Email: ${ }^{*}$ elisabeth.severinsson@hbv.no, anne.holm@hsh.no
}

Received 7 April 2015; accepted 5 May 2015; published 7 May 2015

Copyright (c) 2015 by authors and Scientific Research Publishing Inc.

This work is licensed under the Creative Commons Attribution International License (CC BY). http://creativecommons.org/licenses/by/4.0/

(c) $\underset{\mathrm{EY}}{\mathrm{EY}}$ Open Access

\begin{abstract}
Depression among the elderly is a public health issue. This paper demonstrates the value of patient safety research for future strategies in this area. The aim of the present study was to analyse the relationship between the World Health Organization (WHO) Patient Safety (PS) Model and empirical research on depressed elderly patients' experiences of quality and safe care. The research question was: Which patients' experiences could be linked to quality and safe care as recommended by the WHO? We adopted an implementation approach as the starting point for this interdisciplinary project. A total of 29 individual narrative-based, in-depth interviews were performed to explore patients' experiences and two healthcare teams participated in the focus group interviews. Interpretation of the results revealed that the 23 components of the PS model were linked to elderly patients' experiences of quality and that safe care was not achieved. There was evidence of low quality and lack of safe care due to psychological distress, stress and fatigue, the absence of involvement in decision-making, misdiagnosis, sleep problems as a result of harm from medical error and a poor physical state. Patients' experiences of loneliness gave rise to suicidal thoughts. In conclusion, quality improvement is necessary in all components of the WHO PS model. We recommend structural, process and outcome improvements, more specifically: active involvement, shared decision-making and increased self-management.
\end{abstract}

\section{Keywords}

Chronic Care Management, Implementation, Patient Safety Model, WHO

\footnotetext{
${ }^{*}$ Corresponding author.
}

How to cite this paper: Severinsson, E. and Holm, A.L. (2015) Evaluating Improvement in the Care of Depressed Elderly Patients: An Empirical Approach to the WHO Patient Safety Model. Open Journal of Nursing, 5, 397-406. 


\section{Introduction}

Depression in later life is a major public health issue. Older people are underrepresented in public health research in Europe [1]-[3]. Research on promotion, prevention and policy is scarce compared with research with an epidemiological approach. A sense of safety and security at home is important not only for the depressed elderly patients themselves but also for their family members [4]. Norwegian policy for depressed elderly patients aims to enhance their chances of living an active life in their own home in combination with support from the health and social care services [5] [6].

\subsection{Background}

A large body of literature on evidence-based improvement strategies has been developed to enhance the quality of care and strengthen the safety culture. A working definition of quality consists of clinical effectiveness, patients' safety and patients' experiences [7]. According to Øvretveit [8], evaluation of health interventions, improvements and their implementation is important. Potential health improvements are interventions by individual practitioners or organizations to deliver a better health service to patients [8]. There are several implementation strategies for improving the quality of care and ensuring patient safety [9]. Evidence of the important role of context in patient safety practices has been accumulated ([10], p. 614). Four domains are essential for the implementation of such practices: 1) safety culture, teamwork and leadership; 2) structural and organisational characteristics (e.g. size, organisational complexity and financial status); 3) external factors (e.g. financial or performance incentives and patient safety practice regulations); and 4) availability of implementation and management tools (e.g. training resources or internal organisational incentives) [10]. According to the review of implementation science in the quality and safety domain by Braitwaite et al. [11], the eight success factors are: preparing for change, capacity for implementation—people, capacity for implementation—setting, types of implementation, resources, leverage, desirable implementation and sustainability. Implementation research on patients' experiences of care and safety is scare.

Depression and mental health problems have been described as emotional pain, suffering and loneliness [12]. A systematic review of older patients' narratives about what they need in order to survive when suffering from depression reveals three themes: the need for courage, strength and self-reliance; a struggle to perceive meaning in the meaningless and assuming responsibility [12] [13]. The clinical processes in the healthcare environment are becoming increasingly complex, leading to patient safety risks such as medical errors and complications. Safety is one vital component of high quality care [14].

\subsection{Patient Safety Model}

Safety is defined by the WHO [15] as part of the quality agenda and therefore a dimension of the quality culture. It has also been stated that patient safety is an applied science [16]. The problem of unsafe care has been highlighted by the WHO [3]. The Alliance of Patient Safety commissioned an overview of the research in this area and 23 major patient safety issues were identified, of which eight were classified as structural, five as process and 10 as outcome [17]. Structural factors that contributed to unsafe care were: organizational determinants and latent failures; structural accountability; safety culture; inadequate training and education; stress and fatigue; in addition to production pressure. Knowledge of the role of organizational structure, capacity and communication breakdown in unsafe care is lacking [17]. The key areas of the process domain were misdiagnosis, poor test follow-up, errors in the structure and process, counterfeit and substandard drugs, inadequate patient safety strategies and unsafe injection practice. The safety issues related to patient outcomes were adverse events due to medical devices, medications, surgery, unsafe blood products, falls in hospital and decubitus ulcers. Patient safety among older adults was also a major outcome issue. Such knowledge is important in relation to implementation research.

\subsection{Implementation of the Chronic Disease Management Project}

This study is a part of the extensive project "Chronic Disease Management-Implementation and Coordination of Healthcare Systems for Depressed Elderly Persons" that was developed, implemented and evaluated at Buskerud \& Vestfold University College in 2010 by an interdisciplinary group of experts in the field of healthcare sciences comprising five researchers representing four disciplines [18]-[20]. The central aims of the project 
were: to identify depressed elderly patients' subjective experiences of healthcare systems; to implement the Chronic Care Model (CCM) [21]; and to evaluate the quality of care provided in relation to the determinants in the CCM. The CCM improvement model consists of six interrelated components that are essential for providing quality care to patients with chronic illness: self-management support, clinical information systems, delivery system redesign, decision support, the healthcare organization and the linkages to community-based resources. A definition of implementation research is "to promote the systematic uptake of research findings and other evidence-based practices in routine practice and, hence, to improve the quality and effectiveness of health services” ([22], p. 1). A total of twenty empirical articles based on data from this interdisciplinary research have been published [18]-[20] [23]-[30].

\subsection{Problem Statement}

In the present paper, we aimed to expand our previous research on the delivery of care to depressed elderly patients by interpreting the WHO PS model in relation to empirical outcomes. More specifically, we explored the link to patient safety by means of three quality domains comprising major patient safety issues, i.e., structural, process and outcome [17], assessing the perspectives of both patients and mental healthcare team members as revealed in the published papers. The research question was: Which patients' experiences could be linked to quality and safe care as recommended by the WHO?

\section{Methods}

\subsection{Data Collection}

Data were collected over a four year period (April 2010-May 2014) by the research team in Norway. The participants were recruited by mental health nurses/stakeholders working with the target group. The major area covered in the interviews was; the patients' experiences of quality and safe care. An example of a question is: What does it mean to you to receive support from the healthcare system? As it was expected that some improvement in the quality and safety of care had been achieved, an additional interview was designed to follow up changes in the patients' daily life situation, elaborate on their thoughts of being suicidal, as well as their sense of loneliness and safety. In addition, two mental healthcare teams participated in focus group interviews in order to illuminate their perspective on the quality and safety of care provided to patients. The participants were free to talk about experiences that were important to them. The interviews, which lasted between 60 and 120 minutes, were conducted by two of the researchers, who are mental health nurses with long experience of psychiatric care and research. The second author presented the CCM [21] to specially invited community and specialist healthcare staff in one community during a one and a half hour education session. The second author also conducted two multistage focus group sessions together with a co-moderator, each lasting one and a half to two hours.

\subsection{Participants}

Twenty nine in-depth interviews were initially conducted, followed by an additional ten interviews to deepen understanding of specific suicidal thoughts as well as changes that had taken place during the implementation of the CCM and in the following year. The participants comprised 26 women and three men aged between 60 - 91 years (mean 66 years) from one hospital in a city on the west coast of Norway. The project group had intended to conduct in-depth interviews with 40 patients, but only found 29 who were willing to be interviewed.

The interviews were conducted in the participants' own homes or in the researcher's offices. Five of the participants were married and/or living with a partner, thirteen were widows, nine were divorced, and two had always lived alone. Most had suffered from mental health problems for many years and been diagnosed with a long-term depressive or mood disorder. Thirteen had a history of suicide ideation over the course of the previous 20 - 40 years (Table 1 ).

The first focus group was at the implementation stage. All members of Team A agreed to participate, while one member of Team B declined participation (Table 2). The mental healthcare teams comprised five mental health nurses (Team A), one specialist geriatric nurse and one physiotherapist. At the follow up of evaluation stage the group comprised three mental health nurses (Team A) and one specialist geriatric nurse (Team B, Table 3). 
Table 1. Demographic characteristics of the depressed elderly patients $(n=29)$.

\begin{tabular}{lc}
\hline Age, years & $60-91$ \\
Range & 66 \\
Mean & 26 \\
Female & 3 \\
Male & 5 \\
Partner & 13 \\
Widow & 9 \\
Divorced & 2 \\
Lived alone & 9 \\
Children & 13 \\
Suicidal history & 12 \\
Lived alone & 11 \\
Female & 2 \\
Male & 11 \\
Depressive disorder & 2 \\
Bipolar & 15 \\
Physical health problems and illnesses & 13 \\
Female & 2 \\
Male & \\
Living in their own home receiving on-going formal support from primary health care or & 29 \\
mental health care during the previous six months
\end{tabular}

Table 2. Demographic characteristics of the participants in Team A and Team B-implementation stage.

\begin{tabular}{cccc}
\hline & Members & Years of work experience & Sex \\
Team A & 5 mental health nurses & 20 years & Female \\
Team B & 1 specialist geriatric nurse & 55 & Female \\
& 1 physiotherapist & 5 years & 44 \\
\hline
\end{tabular}

Table 3. Demographic characteristics of the participants in Team A and Team B-evaluation stage.

\begin{tabular}{ccccc}
\hline & Members & Years of work experience & Sex & Mean age \\
Team A & 3 mental health nurses & 20 years working experiences & Female & 52 \\
Team B & 1 specialist geriatric nurse & 15 years working experiences & Female & 55 \\
\hline
\end{tabular}

\subsection{Data Analysis}

The interviews were analysed by means of qualitative thematic analysis inspired by Graneheim and Lundman [31]. This type of analysis describes visible components such as quotations, referred to as the manifest content, as well as the latent content [30]-[34]. A qualitative content analysis [35] was used in a descriptive study of patients' perceptions of the need for improvements in the delivery of healthcare [36]. Each identified topic was reviewed in detail by experts in order to increase understanding of the obstacles to the delivery of safe care. The second step involved the hermeneutic principle of moving from the whole to the parts and back to the whole [37]. The authors reflected on the text and discussed divergent interpretations before finally achieving consensus [23] [26] [29].

\subsection{Ethical Considerations}

Ethical approval was granted by The Regional Ethics Committee of Western Norway (No. 2010/2242). The ethical guidelines specified by the Declaration of Helsinki were applied. The participants were provided with written information and signed a consent form. In addition, they were assured of confidentiality and informed that they could withdraw at any time. The interviews were performed in a sensitive manner and if the topics were too upsetting the participants could discuss the matter with a staff member from the mental health services [38]. The 
interviewers were aware that time was needed for clarification in order to help the participants to understand the questions.

\section{Findings}

Despite indications of positive associations between their experiences and safety, the participants reported several structural components that contributed to unsafe care. The participants' experiences of quality and safe care were at different levels. We found that major contributors to unsafe care were related to organizational determinants such as lack of trust in the commitment of the community healthcare system to bring about effective care [33] [39]. It also concerned structural accountability, which can be counterproductive and have serious implications for patient dignity and safety [30]. The lack of an organizational structure, healthcare professionals' inability to change, communication problems and the absence of collaboration between different healthcare systems were reported [13] [28] [40]. In addition, healthcare professionals did not provide adequate information about the patients' condition and treatment. Nor was the communication between the responsible professionals and the patients satisfactory. The patients reported that healthcare professionals did not always listen to them or take their opinion seriously. We identified a lack of knowledge about how to overcome relational challenges, such as problems associated with communication [36]. It was found that structural issues associated with ethical dilemmas involving attitudes, values and norms were likely to have an impact on safety and the risk of failure [33].

The processes that underlie unsafe care were related to misdiagnosis or not being understood when explaining physical health problems, leading to experiences of stigma and the sense of not being taken seriously or considered deviant [26]. Lack of care also concerned depressed elderly patients' experiences of their physical health problems being affected by stigma, which meant that their need of care was not met and they were not taken seriously, which made them uncertain about whether the pain was physical or mental. They felt dizzy, like a stranger, afraid, helpless and dependent on others [28].

It was stated that failure to adequately follow-up important symptoms constituted a major risk to patient safety. Lack of shared decision-making and user involvement was reported [30]. Another process underlying unsafe care was uncertainty, resulting in fear of becoming dependent on others [26]. In terms of relational aspects, the participants expected emotional and psychological support, relief from fear and anxiety, to be treated with respect, dignity, and compassion, as well as to be understood and listened to [29]. Other aspects identified were the need for clear information and communication that was tailored to patients' needs in order to enable shared decision-making [30].

The reported outcomes of unsafe care included not being understood when presenting with a fractured ankle due to being in a state of psychosis [26] and not being believed when explaining to a GP about the development of a prolapse in the neck because of suffering from major depression [26]. Our data suggest that unsafe care occurs too often, sometimes due to lack of access to appropriate care.

The improvements necessary to ensure quality and safe care were: 1) patient-centred care to enable independence and well-being, 2) greater involvement in decision-making, social participation and activities and 3) redesign of the healthcare organization and safety culture. Overall, the depressed elderly patients' expectations of the healthcare system were not fulfilled. There was no improvement in their situation, which had become even worse in relation to being suicidal. The patients' need to achieve independence and well-being was not met. They wished to be involved in decision-making, become more independent and take part in social activities. Playing an active role in society, being informed, participating, being heard and being autonomous were also highlighted. The necessity of redesigning the healthcare organisation was also mentioned as an important strategy for increasing patient safety.

\section{Discussion}

Improvement in the delivery of safe care to depressed elderly patients was evaluated and linked to the WHO PS model, revealing the need for change. In the future, summative evaluation will be necessary to describe key elements of the complexity of improvement for these patients. We implemented the six CCM components to improve the care, but not all initial implementation goals were achieved. This study demonstrates the difficult challenges involved in integrating health and social care, which is in accordance with Øvretveit et al. [41], who reported that coordinated actions at different levels are required and that a phased approach is necessary when management capacity and outside expertise are limited. 
There is a need for patient-centred care in order to promote independence and well-being among depressed elderly patients. The development of a patient-centred care model that acknowledges an individual patient's needs and values is necessary for improving quality and safe care. Patients should be involved in decisions about their situation and/or treatment and the healthcare services need to be redesigned in order to support self-management. Shared decision making is an important component of a patient-centred care [42]. However, the question of whether patient-centred care leads to better outcomes needs further investigation. Another improvement would be the use of a clear theoretical framework for sharing power and responsibility that links dimensions of patient safety with specific outcomes. Older patients with depression and those who had been treated for a longer period reported far less shared decision making [42]. A study by Holm et al. [30] evaluating healthcare team members' perceptions of their role in encouraging patients to participate in shared decision-making revealed the importance of preventing the violation of human dignity based on changing understanding and attitudes, increasing patients' autonomy and clarifying the team coordinator's role and responsibility. Implementation of shared decision-making improves patients' satisfaction and reduces the imbalance of power between healthcare professionals and patients [43]. The involvement of mental healthcare patients in decision-making processes is considered an ethical requirement [44] but demands careful management to realise its full potential [45]. Being active and developing a network are important for the patients' well-being when suffering from mental health problems. Interdisciplinary leadership also appears to be necessary for overcoming difficulties and improving health behaviours that impact on quality of life.

It is essential to increase patients' participation in the provision of safe care. This research has focused on depressed elderly patients, who are a vulnerable population in terms of adverse events. A review conducted to delineate factors that could be of importance for patient participation in quality and safety issues revealed five broad categories [46]. Two of them are relevant when interpreting the findings of this study. According to Davis et al. ([46], p. 260), factors that could affect patient participation in their care are: patient-related (i.e., patients' knowledge and beliefs about safety, emotional experiences of healthcare delivery and relevant coping styles; and demographic characteristics) and illness-related (i.e., the stage and the severity of patients' illness(es); as well as their symptoms, treatment plan, and experiences of illness).

Interpretation of the WHO's major patient safety issues was empirically evaluated. On the structural level, several aspects of organisational determinants and latent failures, lack of appropriate knowledge, availability of knowledge and knowledge transfer, as well as an inadequate number of qualified healthcare professionals were reported, resulting in communication problems and stress [32]. Regarding the process factor quality domain, errors were reported in the care process due to misdiagnosis, lack of adequate follow-up, unsafe care and counterfeit drugs [18]. Patient safety topics described in the quality domain related to the outcomes of unsafe care were adverse events due to medication and the absence of the patients' voices [18].

Increasing the use of evidence-based practice could potentially reduce unsafe care [17]. Bishop and Macdonald [47] revealed four themes; wanting control, feeling connected, encountering roadblocks and sharing responsibility for safety. They concluded that current strategies aimed at increasing patient awareness of patient safety may not be enough and that targeted interventions to strengthen patient control should therefore be developed. Patient safety is high on the priority list of international organisations. A systematic review of the evidence demonstrated positive associations between patients' experience and two domains of quality, i.e., safety and effectiveness [48]. Patients' experience is recognized as one of the three pillars of quality in healthcare alongside effectiveness and patient safety. Teamwork and leadership are core components in the provision of quality care and thus influence the improvement of patient safety practices [49].

Building capacity in patient safety, quality improvement and innovation in healthcare is necessary. It is surprising that patients are not involved in shared decision making. The findings from this study can be explained in accordance with the research by Davies et al. ([50], p. 174) on staff members' and patients' attitudes towards patient involvement. The researchers recommend fostering relationships between patients and healthcare professionals as a means of creating an environment where patient participation is valued and supported, thus making patient involvement in promoting safety a working reality. The differences in the attitudes of healthcare professionals and patients have been studied by Davis et al. [51]. Healthcare professionals attitudes were affected by their own behaviour, who the healthcare professional was interacting with and her/his own professional role. From the patients' perspective, quality included access to care, responsiveness and empathy, good communication, clear information, appropriate treatment, relief of symptoms, improvement in health status and above all safety and freedom from medical injury [52]. Thus the active role of patients in their care should be recognised 
and focused on in healthcare. However, not all patients want to be actively involved. An explorative study was conducted by Davis et al. [53] to answer the question: How willing are patients to question healthcare staff on issues related to the quality and safety of their healthcare in a surgical setting? Characteristics of the surgical patient who did not want to be involved were male, lower educational level and unemployed. The authors concluded that patient involvement strategies that take patient characteristics into account need to be developed for patients and staff in order to promote patient participation. The empirical approach in this study was to evaluate depressed elderly patients. Optimizing patient involvement in quality improvement in mental health is important. Barriers to and facilitators of involvement in mental health research were investigated by Ennis and Wykes [54], who revealed that patient involvement increased over time although it was limited in some research areas. The authors recommend researchers to involve patients to a greater degree, as this is associated with successful results.

A limitation of this study is that the data collection only took place in one region. An additional limitation is the small sample, comprising seven healthcare professionals in the first focus group and four in the second. Due to these limitations the results are only valid for the study group.

\section{Conclusion}

Research focusing on patients' experiences of quality and safe care will improve care. The evidence in the present study demonstrates some positive associations. However, there are also indications that patients are dissatisfied with the care due to lack of information and poor communication. Patient involvement such as shared decision-making will increase the quality and safety of care.

\section{Acknowledgements}

We wish to express our appreciation to the patients, the healthcare team members and members of the research team who participated in the various data collection stages in the research process. We would also like to thank Gullvi Nilsson and Monique Federsel for reviewing the English language. This work was undertaken as a part of the project "Chronic Disease Management-Implementation and coordination of healthcare systems for depressed elderly persons” project funded by the Research Council of Norway (Grant No. 204238/V50) from 2010-2014.

\section{Contributors}

The study was designed by ES and ALH. ES coordinated the research. ALH and ES participated in the data analysis and interpretation. The report was written by ES and ALH.

\section{References}

[1] World Health Organization (2005) Mental Health Action Plan for Europe, Facing Challenges, Building Solutions. Reports of the WHO European Ministerial Conference on Mental Health, Helsinki.

[2] World Health Organization (2008) Primary Health Care—Now More than Ever. http://www.who.int/whr/2008/whr08_en.pdf?ua=1

[3] World Health Organization (2012) Mental Health. Depression. World Health Organization. http://www.who.int/mental_health/management/depression/en/

[4] European Commission (2005) Improving the Mental Health of the Population: Towards a Strategy on Mental Health for the European Union. Green Paper COM, (2005) 484, EU Health and Consumer Protection Directorate-General, Brussels.

[5] The Norwegian Ministry of Health \& Care Services (2009) The Coordination Reform. Proper Treatment-At the Right Place and Right Time. Report 47 to the Storting Oslo, Norway.

[6] The Norwegian Ministry of Social and Health Affairs (1999) National Action Programme for Mental Health. Norwegian Ministry of Social and Health Affairs, Oslo.

[7] Robert, G.B., Anderson, J.E., Burnett, S.J., Aase, K., Andersson-Gare, B., Bal, R., Calltorp, J., Nunes, F., Weggelaar, A.-M., Vincent, C.A. and Fulop, N. (2011) A Longitudinal, Multi-Level Comparative Study of Quality and Safety in European Hospitals: The QUASER Study Protocol. BMC Health Services Research, 11, 285.

http://biomedcentral.com/1472-6963/11/285

http://dx.doi.org/10.1186/1472-6963-11-285 
[8] Øvretveit, J. (2014) Evaluating Improvement and Implementation for Health. Open University Press, New York.

[9] Severinsson, E. (2014) Effectiveness and Implementation of Patient Safety Care. Journal of Nursing Management, 22, 823-824. http://dx.doi.org/10.1111/jonm.12263

[10] Taylor, S., Dy, S., Foy, R., Hempel, S., McDonald, K.M., Øvretveit, J., Pronovost, P., Rubenstein, L., Wachter, R. and Shekelle, P. (2011) What Context Feature Might Be Important Determinants of the Effectiveness of Patient Safety Practice Interventions? BMJ Quality Safety, 20, 611-617. http://dx.doi.org/10.1136/bmjqs.2010.049379

[11] Braitewaite, J., Marks, D. and Taylor, N. (2014) Harnessing Implementation Science to Improve Care Quality and Patient Safety: A Systematic Review of Targeted Literature. International Journal for Quality in Health Care, 26, 321329. http://dx.doi.org/10.1093/intqhc/mzu047

[12] Holm, A.L. and Severinsson, E. (2014) Surviving Depressive Ill-Health: A Qualitative Systematic Review of Older Persons’ Narratives. Nursing \& Health Sciences, 16, 131-140. http://dx.doi.org/10.1111/nhs.12071

[13] Holm, A.L. and Severinsson, E. (2012) Systematic Review of the Emotional State and Self-Management of Widows. Nursing and Health Sciences, 14, 109-120. http://dx.doi.org/10.1111/j.1442-2018.2011.00656.x

[14] Corrigan, J. (2005) Crossing the Quality Chasm. In: Proctor, R., Compton, D., Grossman, J. and Fanjiang, G., Eds., Building a Better Delivery System: A New Engineering/Health Care Partnership, The National Academies, Washington DC.

[15] WHO (2009) More than Words. Conceptual Framework for the International Classification for Patient Safety. http://www.who.int/patientsafety/implementation/taxonomy/icps technical report en.pdf

[16] Pronovost, P.J., Goeschel, C.A., Marsteller, J.A., Sexton, J.B., Pham J.C. and Berenholtz, S.M. (2009) Framework for Patient Safety Research and Improvement. Circulation, 119, 330-337. http://dx.doi.org/10.1161/CIRCULATIONAHA.107.729848

[17] Jha, A.K., Prasopa-Plaizier, N., Larizgoitia, I. and Bates, D.W. (2010) Patient Safety Research: An Overview of the Global Evidence. Quality Safe Health Care, 19, 42-47. http://dx.doi.org/10.1136/qshc.2008.029165

[18] Severinsson, E. and Holm, A.L. (2014) Understanding and Improving the Care in the Context of Depressed Elderly Person Living in Norway. Open Journal of Nursing, 4, 809-817. http://dx.doi.org/10.4236/ojn.2014.412086

[19] Severinsson, E. and Holm, A.L. (2014) Implementation Strategies for Improving the Care of Depressed Elderly Persons-Summary of an International Workshop. Open Journal of Nursing, 4, 715-723.

[20] Severinsson, E. and Holm, A.L. (2014) Chronic Disease Management: Implementation and Coordination of Healthcare Systems for Depressed Elderly Persons. Issues of Mental Health Nursing, 35, 934-939. http://dx.doi.org/10.3109/01612840.2014.930215

[21] Wagner, E.H., Austin, B.T., Davis, C., Hindmarsh, M., Schaefer, J. and Bonomi, A. (2001) Improving Chronic Illness Care: Translating Evidence into Action. Interventions That Encourage People to Acquire Self-Management Skills Are Essential in Chronic Illness Care. Health Affairs, 20, 64-78. http://dx.doi.org/10.1377/hlthaff.20.6.64

[22] Eccles, M.P. and Mittman, B.S. (2006) Welcome to Implementation Science. http://www.implementationscience.com/content/1/1/1

[23] Holm, A.L., Lyberg, A., Berggren, I., Lassenius, E. and Severinsson, E. (2013) Older Persons’ Lived Experiences of Depression and Self-Management. Issues in Mental Health, 34, 757-764. http://dx.doi.org/10.3109/01612840.2013.809829

[24] Holm, A.L. and Severinsson, E. (2013) A Qualitative Systematic Review of Older Persons' Perceptions of Health, Ill Health and Their Community Health Care Needs. Nursing Research \& Practice, 2013, Article ID: 672702. http://dx.doi.org/10.1155/2013/672702

[25] Holm, A.L. and Severinsson, E. (2014) Perceptions of the Need for Improvements in Healthcare after Implementation of the Chronic Care Model. Nursing \& Health Sciences, 16, 442-448. http://dx.doi.org/10.1111/nhs.12136

[26] Holm, A.L., Lyberg, A. and Severinsson, E. (2014) Living with Stigma: Depressed Elderly Persons’ Experiences of Physical Health Problems. Nursing Research \& Practice, 2014, Article ID: 527920. http://dx.doi.org/10.1155/2014/527920

[27] Holm, A.L. and Severinsson, E. (2013) Interdisciplinary Team Collaboration during Discharge of Depressed Older Persons: A Norwegian Qualitative Implementation Study. Nursing Research \& Practice, 2013, Article ID: 794743. http://dx.doi.org/10.1155/2013/794743

[28] Holm, A.L., Lyberg, A., Berggren, I. and Severinsson E. (2014) Going Around in a Circle: A Norwegian Study of Suicidal Experiences in Old Age. Nursing Research \& Practice, 2014, Article ID: 734635.

[29] Lyberg, A., Holm, A.L., Lassenius, E., Berggren, I. and Severinsson, E. (2013) Older Persons' Experiences of Depressive Ill-Health and Family Support. Nursing Research \& Practice, 2013, Article ID: 837529. http://dx.doi.org/10.1155/2013/837529 
[30] Holm, A.L., Lyberg, A., Berggren, I. and Severinsson, E. (2014) Shared Decision-Making after Implementation of the Chronic Care Model (CCM) —An Evaluative Approach. Open Journal of Nursing, 4, 824-835. http://dx.doi.org/10.4236/ojn.2014.412088

[31] Graneheim, U.H. and Lundman, B. (2004) Qualitative Content Analysis in Nursing Research: Concepts, Procedures and Measures to Achieve Trustworthiness. Nurse Education Today, 24, 105-112. http://dx.doi.org/10.1016/j.nedt.2003.10.001

[32] Holm, A.L. and Severinsson, E. (2013) Interdisciplinary Team Collaboration during Discharge of Depressed Older Persons: A Norwegian Qualitative Implementation study. Nursing Research and Practice, 2013, Article ID: 794743.

[33] Holm, A.L. and Severinsson, E. (2014) Reflection on the Ethical Dilemmas Involved in Promoting Self-Management. Nursing Ethics, 21, 402-413. http://dx.doi.org/10.1177/0969733013500806

[34] Holm, A.L., Lyberg, A., Berggren, I., Cutcliffe, J. and Severinsson, E. (2014) Shadows from the Past. The Situated Meanings of Being Suicidal among Depressed Older People Living in the Community. Crisis, 35, 253-260. http://dx.doi.org/10.1027/0227-5910/a000259

[35] Sandelowski, M. (2001) Focus on Research Methods. Whatever Happened to Qualitative Description? Research in Nursing Health, 23, 334-340. http://dx.doi.org/10.1002/1098-240X(200008)23:4<334::AID-NUR9>3.0.CO;2-G

[36] Holm, A.L., Lyberg, A., Berggren, I., Åström, S. and Severinsson, E. (2014) Social Capital and Depressive IllHealth-An Evaluative Approach to the Implementation of the Chronic Care Model (CCM). Open Journal of Nursing, 4, 683-694. http://dx.doi.org/10.4236/ojn.2014.410073

[37] Gadamer, H.G. (2004) Truth and Methods. Interactive Science, Gloucester.

[38] Liamputtong, P. (2007) Researching the Vulnerable: A Guide to Sensitive Research Methods. Thousand Oaks, London.

[39] Holm, A.L. and Severinsson, E. (2012) Chronic Care Model for the Management of Depression: Synthesis of Barriers to, and Facilitators of, Success. International Journal of Mental Health Nursing, 26, 513-523. http://dx.doi.org/10.1111/j.1447-0349.2012.00827.x

[40] Holm, A.L. and Severinsson, E. (2014) Effective Leadership of Older Persons in the Community-A Systematic Review. Journal of Nursing Management, 22, 211-224. http://dx.doi.org/10.1111/jonm.12076

[41] Øvretveit, J., Hansson, J. and Brommels, M. (2010) An Integrated Health and Social Care Organisation in Sweden: Creation and Structure of a Unique Local Public Health and Social Care System. Health Policy, 97, 113-121. http://dx.doi.org/10.1016/j.healthpol.2010.05.012

[42] Solberg, L.I. Crain, L., Rubenstein, L., Unützer, J., Whitebird, R.R. and Beck, A. (2014) How Much Shared Decision Making Occurs in Usual Primary Care of Depression? Journal of the American Board of Family Medicine, 27, 199-208. http://dx.doi.org/10.3122/jabfm.2014.02.130164

[43] Dierkx, K., Deveugele, M., Roosen, P. and Devish, I. (2013) Implementation of Shared Decision Making in Physical Therapy: Observed Level of Involvement and Patient Preference. Physical Therapy, 93, 1321-1330. http://dx.doi.org/10.2522/ptj.20120286

[44] Tambuyzer, E., Pieters, G. and Van Audenhove, C. (2011) Patient Involvement in Mental Healthcare: One Size Does Not Fit All. Health Expectations, 17, 138-150.

[45] Armstrong, N., Herbert, G., Aveling, E.L., Dixon-Woods, M. and Martin, G. (2013) Optimizing Patient Involvement in Quality Improvement. Health Expectations, 16, e36-e47. http://dx.doi.org/10.1111/hex.12039

[46] Davis, R.E., Jacklin, R., Sevdalis, N. and Vincent, C. (2007) Patient Involvement in Patient Safety: What Factors Influence Patient Participation and Engagement? Health Expectations, 10, 259-267. http://dx.doi.org/10.1111/j.1369-7625.2007.00450.x

[47] Bishop, A.C. and Macdonald, M. (2014) Patient Involvement in Patients Safety: A Qualitative Study of Nursing Staff and Patient Perceptions. Journal of Patient Safety, in Press. http://dx.doi.org/10.1097/PTS.0000000000000123

[48] Doyle, C., Lennox, L. and Bell, D. (2013) A Systematic Review of Evidence on the Links between Patient Experience and Clinical Safety and Effectiveness. BMJ Open, 3, e001570. http://dx.doi.org/10.1136/bmjopen-2012-001570

[49] Severinsson, E. (2013) Patient Safety Management. What Do the Patients Want? Journal of Nursing Management, 21, 203-205. http://dx.doi.org/10.1111/jonm.12074

[50] Davis, R.E., Sevdalis, N., Pinto, A., Darzi, A. and Vincent, C. (2011) Patients’ Attitudes towards Patient Involvement in Safety Interventions: Results of Two Exploratory Studies. Health Expectation, 16, e164-e176. http://dx.doi.org/10.1111/j.1369-7625.2011.00725.x

[51] Davis, R.E., Sevdalis, N. and Vincent, C. (2012) Patient Involvement in Patient Safety: The Healthcare Professional's Perspective. Journal of Patient Safety, 8, 182-188. http://dx.doi.org/10.1097/PTS.0b013e318267c4aa

[52] Vincent, C.A. and Coulter, A. (2002) Patient Safety: What about the Patient? www.qualityhealthcare.com 
[53] Davis, R.E., Koutantji, M. and Vincent, C.A. (2008) How Willing Are Patient to Question Healthcare Staff on Issues Related to the Quality and Safety of Their Healthcare? An Exploratory Study. Quality Safe Health Care, 17, 90-96. http://dx.doi.org/10.1136/qshc.2007.023754

[54] Ennis, L. and Wykes, T. (2013) Impact of Patient Involvement in Mental Health Research: Longitudinal Study. The British Journal of Psychiatry, 203, 381-386. http://dx.doi.org/10.1192/bjp.bp.112.119818 